\title{
Contamination potentielle des aliments par des polychlorobiphényles (PCBs): Connaissance du polluant et évaluation de la perception du risque
}

\author{
M. Mani Oumarou TIDJANI ${ }^{1}$, Amadou ISSOUFOU ${ }^{2 *}$, \\ Adamou RABANI ${ }^{1}$ et $\mathrm{S}$. Salamou ZEINAB ${ }^{3}$ \\ ${ }^{I}$ Departement de Chimie, Faculté des Sciences et Techniques, Université Abdou Moumouni de Niamey, Niger. \\ ${ }^{2}$ Département des Sciences Fondamentales et d'Ingénierie Biologique, Faculté d'Agronomie et des Sciences de \\ l'Environnement, Université Dan Dicko Dankoulodo de Maradi, BP 465 ADS Maradi, Niger. \\ ${ }^{3}$ Institut Bouzou Dan Zambadi, Université Libre de Maradi, Niger. \\ *Auteur correspondant ; E-mail: issoufsara@gmail.com; issoufou.amadou@uddm.edu.ne ; \\ Tel.: +227-2041-0132; Fax: +227-2041-0133
}

\section{RESUME}

Les différentes voies d'exposition aux polluants récalcitrants, comme les PCBs, pour l'être humain sont, en plus de denrées aliments contaminés, la respiration d'air contaminé à proximité des transformateurs électriques en fuite ou usagés, contenant ou contaminés aux PCBs. Cela pourrait être, en effet, le cas dans la plupart des zones urbaines au Niger. Pour vérifier le niveau d'information et de connaissance du polluant par la population et évaluer la perception des risques liés aux PCBs, il a été entrepris une investigation. Les informations ont été recueillies par la méthode des questionnaires aux quelles une centaine de répondants de la population urbaine de Maradi ont été soumis. Il en résulte que $23 \%$ seulement de la population enquêtée ont la notion de la pollution et de l'exposition aux PCBs, lorsque $77 \%$ n'ont pas conscience de cette situation de risque, alors qu'en termes de la perception des risques, les $31 \%$ de ceux qui ont cette notion, sont capables d'établir le lien du transfert des PCBs de l'environnement vers l'homme directement, ou via l'alimentation. Enfin, les résultats de ce type d'enquête pourraient servir à la mise en place d'une stratégie de prévention et de protection de la population contre les polluants dangereux.

(C) 2018 International Formulae Group. All rights reserved.

Mots clés. Huile de transformateur, environnement, air, produits alimentaires, exposition, danger.

\section{Potential contamination of food with polychlorinated biphenyls (PCBs): Knowledge of pollutant and assessment of risk perception}

\begin{abstract}
The different ways of exposure to recalcitrant pollutants, such as PCBs, for humans are contaminated foodstuffs, the breathing of contaminated air in the vicinity of leaking or used electrical transformers, containing or contaminated with PCBs. This could be the case in most of the urban areas in Niger. To verify the level of information and knowledge of the pollutant by the population and to assess the perception of risks related to PCBs; a survey was carried out. The information was collected by the questionnaire method, using a hundred of respondents from the urban population of Maradi. The result showed that only $23 \%$ of the
\end{abstract}


population surveyed have the basic knowledge of pollution and exposure to PCBs, while $77 \%$ were not aware of this risk. Whereas, in terms of risk perception $31 \%$ of those who have this concept were able to establish the link between the transfer of PCBs to human through environment, or via the foods. In conclusion, these results could be used for the implementation of a strategy for the prevention and protection of the population against harmful pollutants.

(C) 2018 International Formulae Group. All rights reserved.

Keywords : Transformer oil, environnement, air, foodstuffs, exposition, danger.

\section{INTRODUCTION}

Dans un contexte où se pose avec acuité la gestion des déchets industriels et des déchets de consommation pour débarrasser l'environnement des substances polluantes de plus en plus toxiques (Nwinyi, 2011), il s'impose une exigence de la qualité alimentaire, nonobstant une forte contamination de toutes les matrices environnementales (Sopaj, 2013). En effet, une forte contamination des écosystèmes et de nombreux êtres vivants a entraîné une exposition permanente des aliments et de plusieurs espèces $y$ compris l'homme (Bodiguel, 2008). Certains de ces contaminants sont rémanents, et les plus dangereux d'entre eux sont les polluants organiques persistants, dont les polychlorobiphényles appelés communément PCBs (Li, 2007). Les polychlorobiphényles sont des composés aromatiques chlorés, constitué d'un noyau biphényle $\left(\mathrm{C}_{6} \mathrm{H}_{5}-\mathrm{C}_{6} \mathrm{H}_{5}\right)$ où un ou plusieurs atomes d'hydrogènes ont été substitués par ceux de chlores. Le degré $n$ de substitution pouvant varier de 1à 10, les PCBs ont pour formule générale $\mathrm{C}_{6} \mathrm{H}_{10-\mathrm{n}} \mathrm{Cl}_{\mathrm{n}}$. Ils sont thermiquement et chimiquement très stables; ce qui leur a valu une large utilisation dans divers domaines d'activités économiques, particulièrement comme fluide diélectriques dans les transformateurs et condensateurs électriques, avant d'être interdits vers la fin des années 80 (Ayandele et al., 2012). Toutefois, sur les 1.5 millions de tonnes des PCB produites dans le monde entre 1929 et 1989 (Tajik et al., 2012) une grande quantité des PCBs est disséminée dans la nature, ou se retrouvent encore dans des anciens transformateurs électriques en service ou usagés, contaminant ainsi l'eau, l'air, le sol, donc jusqu'aux denrées alimentaires d'origine diverse (Oziolor et al., 2018). Quoique la contamination par les PCBs soit de faible niveau, elle est omniprésente (Imbeault et al., 2000; Weber et al., 2017). Ainsi, les principales voies d'exposition aux PCBs pour l'être humain sont le poisson, la viande, les produits laitiers, les œufs, la respiration d'air contaminé par les PCBs à proximité des vieux transformateurs dans des vieilles installations électriques, des sites d'enfouissement ou de stockages provisoires ne répondant pas aux normes environnementales (Hadnagy et al., 2007).

La toxicité des PCBs et son effet nocif sur la santé humaine sont désormais une évidence à laquelle s'ajoute une cancérogénicité potentielle liée au facteur de bioaccumulation (Mei-Lin et al., 2000; Kerchich et al., 2018). Ce sont en effet, les caractères hydrophobe et liposoluble qui en font des PCBs un contaminant bioaccumulable. Avec une concentration de $8 \times 10^{-12}$ g par gramme de poids frais $\left(8 \mathrm{pg} \mathrm{g}^{-1}\right)$ autorisée pour la consommation humaine (TEQ, règlement CE199/2016); Cette concentration peut être multipliée d'environ 40 fois dans certaines espèces de poisson d'eau douce (313 pg.g ${ }^{-1}$ poids frais TEQ (toxicité équivalente dioxine (TEQ)) pour une espèce de chevaine (Babut et Miège, 2007).

Au Niger, récemment, en début 2017, une importante quantité des déchets des PCBs liquides, du sable et objets souillés par ce liquide ont été exportés du territoire (pour élimination), dans le cadre des activités du projet de suivi de la mise en œuvre de la convention de Stockholm (Direction de l'Environnement, 2017). Malgré cela, il existe encore, beaucoup de déchets des PCBs, 
stockés souvent dans des conditions ne répondant pas aux normes environnementales.

Pour vérifier cela et mesurer le degré d'exposition, et d'une probable contamination de la population, il a été entrepris une investigation à travers un échantillon d'une population urbaine de 100 répondants. Il ressort de cette enquête qu'une grande partie de la population enquêtée n'est pas informée du risque sur la santé humaine et l'effet nocif sur l'environnement. Mieux, certaines personnes interrogées n'ont aucune notion sur les substances polluantes, en particulier les PCBs. Toutefois, ils peuvent témoigner d'une fuite de transformateur ou même de son explosion contaminant l'environnement immédiat, souvent jusqu'aux denrées alimentaires ayant des grandes surfaces de contact. Les résultats de ces travaux quasi inédits permettront de mesurer la notion qu'a la population sur les micropolluants comme les PCBs, et de faire des suggestions aux services en charge de la gestion des produits dangereux afin d'asseoir une stratégie de prévention de la contamination et de la protection de la population.

L'objectif principal de ce travail est de vérifier l'exposition et la probable contamination de l'homme et des aliments qui peuvent se trouver proche d'une source d'émission des PCBs. Pour y parvenir, il s'impose de mesurer d'abord le niveau d'information et de connaissance du polluant PCBs par la population, puis analyser le degré d'exposition et d'évaluer la perception des risques liés aux PCBs qu'a la population.

\section{MATERIEL ET METHODES}

\section{Présentation du cadre d'étude}

Cette étude s'est déroulée dans la ville de Mardi, chef-lieu de la région de Maradi. Elle est située dans le centre Sud du Niger (pays vaste de $1267000 \mathrm{~km}^{2}$ ), entre le $13^{\circ}$ et $15^{\circ} 26^{\prime}$ de latitude Nord et le $16^{\circ} 16^{\prime}$ et $8^{\circ} 36^{\prime}$ de longitude Est. Elle couvre une superficie de $41795 \mathrm{~km}^{2}$ et est limitée au sud par la république fédérale du Nigeria sur environ $150 \mathrm{~km}$ de frontière foreuse. La région a un réseau hydrographique riche et varié avec le lac de Madarounfa comme le point d'eau le plus important de la région. Quant aux sols, ils sont marqués par leur caractère dunaire et peuvent être repartis en sableux, sablodunaires, sablo-argileux et alluvial.

\section{Caractérisation de la population enquêtée}

La population enquêtée est essentiellement urbaine. Puisqu' il s'agit d'une évaluation du risque à partir d'abord de la notion de la pollution, de l'exposition et de la contamination des aliments; la population enquêtée a été groupée en groupe d'âge, classée par niveau d'instruction (degré d'instruction) et répartie en population générale et population professionnelle.

\section{Méthodologie utilisée \\ Collecte des données}

Eu égard à la loi normale, la taille minimum d'un échantillon est en général de 30 individus (Francis et al., 2010). Les données ont été collectées à partir des questionnaires administrées à chacun de cent individus de l'échantillon constitué d'une population générale prise au hasard et d'une population professionnelle souvent en contact direct avec des matériels contenants des PCBs.

Les questionnaires contiennent tout d'abord des interrogations relatives à l'âge et au niveau d'instruction, qui ont permis de regrouper en âge et de classer en fonction du niveau d'instruction les différents répondants. C'est ainsi qu'un premier dépouillement a permis de regrouper les personnes interrogées en cinq groupe d'âges. Le groupe 1 regroupant les individus âgés de moins de 21 ans [âge $\leq 20]$, le groupe 2 les individus dont l'âge est compris entre 21 et 25 ans [21-24], Le groupe 3 de 25 à 39 ans, le groupe 4 de 40 à 49 ans et le groupe 5 constitué des individus dont l'âge est supérieur ou égal à 50 ans.

Le niveau d'instruction a été subdivisé en 8 degrés en l'occurrence : le degré 1 pour une personne ayant été à l'école, mais qui n'a pas bouclé le cycle de l'école primaire (primaire incomplet), le degré 2 pour le primaire complet, le degré 3 pour le secondaire incomplet, le degré 4 pour le 
secondaire complet, le degré 5 pour le Lycée incomplet, 6 pour le Lycée complet, et le degré 7 pour les études supérieures, tandis que le degré 8 est attribué à toute personne instruite pas dans le même système classique subdivisé ci-dessus ; ou dont le métier exercé n'a pas nécessité une formation classique (Figure 1).

\section{Les variables ou paramètres quantifiables}

- La notion de la pollution et information générale sur les PCBs: Ce paramètre a permis de savoir si la population a, d'abord la notion de la pollution et possède une information d'ordre générale sur les PCBs et sait où se situe la source d'émission la plus probable des PCBs actuellement. Pour rendre l'information plus concrète, il a fallu se confiner autour des huiles de transformateurs électriques contenant ou contaminés aux PCBs.

- La notion de la pollution et l'exposition des aliments aux PCBs: La question de la pollution permet d'aller du général, en ce qui concerne les substances polluantes, pour arriver à se poser spécifiquement la question, si les aliments d'origine diverse peuvent être exposés aux PCBs, lorsqu'ils se trouvent dans l'environnement immédiat d'une source probable d'émission de ces substances.

- La notion de la contamination des aliments ou des personnes et des risques liées aux PCBs : La contamination de toutes les matrices de l'environnement est omniprésente. Des très petites quantités des PCBs ont été retrouvées dans les aliments d'origine animales et végétales (à des doses inférieures à la dose admissible dans la plus part des cas) (Andric et al., 2000; Bodiguel, 2008). Il s'agit ici de recueillir des informations sur une probable contamination consécutive à une exposition prolongée (transformateur électrique en fuite) ou chronique lors des accidents d'explosion des transformateurs électriques.

\section{Analyses statistiques}

Les données recueillies après l'enquête ont été visualisées dans les feuilles de calcul à l'aide de logiciel Microsoft office Excel version 2013 où elles ont été analysées. Les différents calculs statistiques (les sommes, les moyennes et fréquences) et les tris ont été faits.
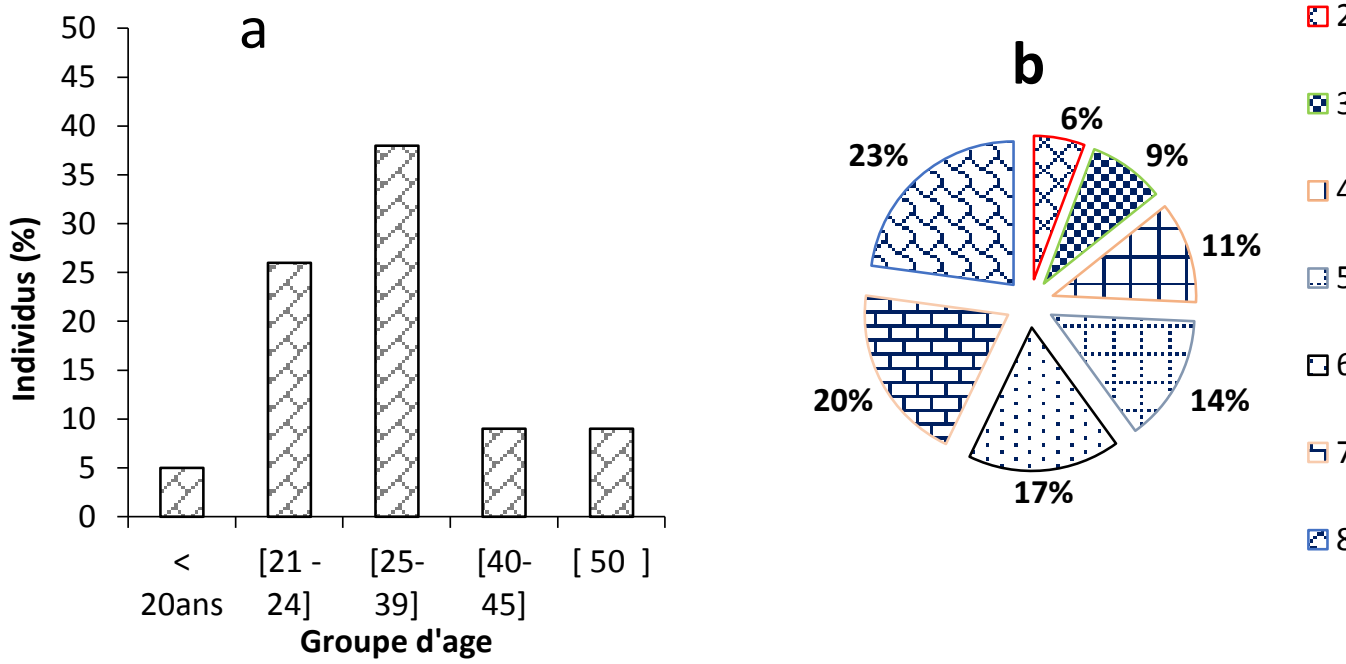

Figure 1: (a) Répartition en âge de la population enquêtée et (b) en fonction du niveau d'instruction. 


\section{RESULTATS}

La Figure 2 présente un transformateur électrique usagé et des futs servant au stockage des déchets solides souillés au pyralène, ce qui traduit le lien réducteur de la source d'émission des PCBs. Sur la Figure 3a, il est montré que $62 \%$ de la population ont répondu parce qu'ils ont une notion de la pollution et ont une information superficielle sur les PCBs. Quant à la Figure 3b, elle précise que $48 \%$ de ceux qui ont cette notion sont âgés de 25 à 39 ans lorsque seulement $7 \%$ et $10 \%$ ont respectivement plus de 50 ans et moins de 20 ans. La Figure 3c indique que $58 \%$ de cette même proportion ont fait des études supérieures contre $5 \%$ de ceux qui ont le niveau du collège. Les résultats relatifs à la notion de la pollution et de l'exposition des aliments aux PCBs sont présentés, d'abord par la Figure 4a qui montre que seulement $23 \%$ de ceux qui ont la notion de la pollution, peuvent aussi appréhender l'exposition des aliments et de l'Homme aux PCBs, alors que les $77 \%$ ne sont pas conscients de cette situation de risque. Mais la Figure $4 \mathrm{~b}$ présente ce constat d'exposition et de la perception du risque en fonction du groupe d'âge, ce qui fait ressortir que $50 \%$ de ceux qui peuvent appréhender l'exposition sont âgés de 25 à 39 ans. C'est aussi le même groupe d'âge qui perçoit mieux le risque $(48 \%)$ de la même manière qu'il peut constater l'exposition. À l'opposé, ce sont les enquêtés de moins de 20 ans (5\% environ) et de plus de 50 ans (10\%) qui appréhendent peu l'exposition et ne perçoivent pas assez le risque. La Figure 5 montre le constat d'exposition aux PCBs et la perception du risque lié aux PCBs en fonction du niveau d'instruction. Sur la même Figure 5, 51\% de ceux qui appréhendent l'exposition aux PCBs, ont un niveau d'instruction de degré 7 (études supérieure) et ne font aucun lien quant aux risques $(0 \%)$ liés aux PCBs. Cette situation n'est pas la même chez les individus au degré 8 d'instruction qui appréhendent l'exposition (40\%) et perçoivent mieux les risques liés aux PCBs (50\%). Les enquêtés qui ont le niveau d'instruction $2,3,4,5$ et 6 n'arrivent pratiquement pas à constater l'exposition et ne perçoivent pas aussi les risques liés aux PCBs. Enfin, la Figure 6 présente la synthèse des paramètres étudiés en indice de risque (indice 0 à indice 5), Ainsi, plus l'indice est élevé plus le répondant a une notion de la pollution et est capable d'appréhender l'exposition aux PCBs. Il fait également la notion d'une probable contamination et a une idée des effets nocifs de ce polluant sur la santé. En somme, cette Figure 6 montre que $31 \%$ (indice 5) de ceux qui ont une information d'ordre générale sur les PCBs sont capables d'établir le lien de transfert de ce polluant vers les êtres vivants, en indiquant sans grande précision les conséquences néfastes. A l'opposé, les 12\% (indice 0 ) ne cernent pas c'est qu'une pollution ni moins l'exposition et n'ont aucune information sur les PCBs afin d'avoir l'idée de la perception du risque. Enfin, ceux qui établissent le lien entre le transfert des PCBs de l'environnement vers l'Homme directement ou via l'alimentation, en indiquant au moins un risque lié à cette contamination, représentent $31 \%$ de la population. 


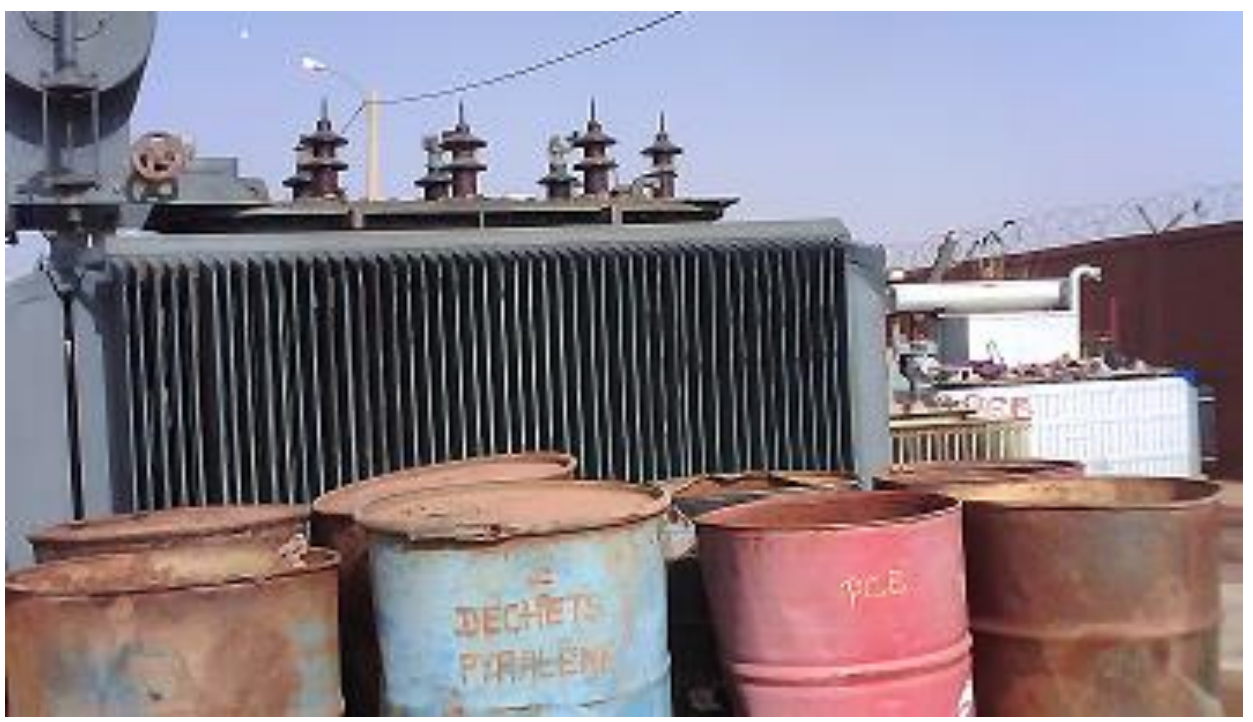

Figure 2: Exemples d'un transformateur usagé et des fûts servant au stockage des déchets solides souillés au pyralène.
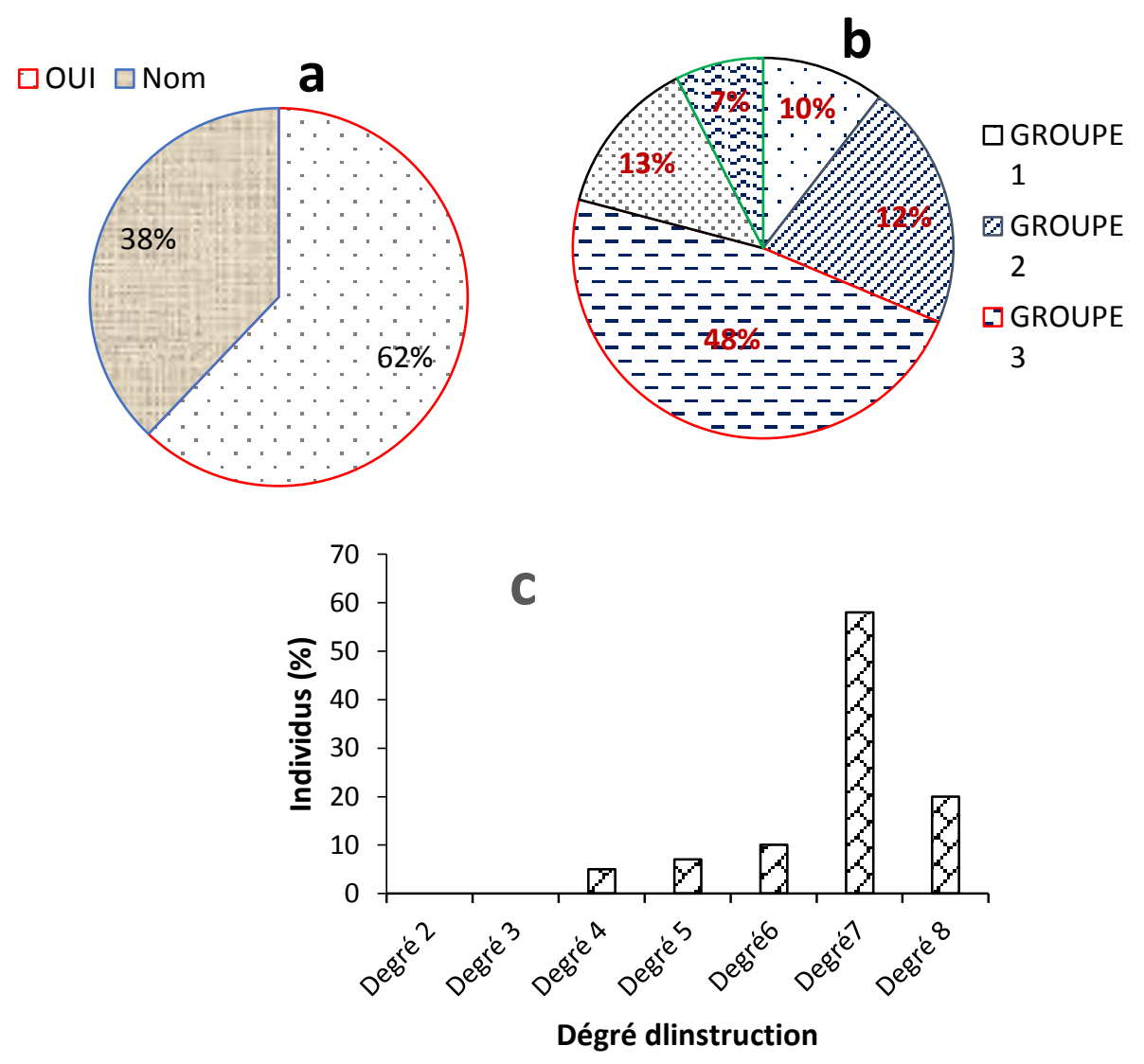

Figure 3: (a) Information générale sur les PCBs, (b) Notion de pollution et d'information sur le PCBs en fonction de groupe d'âge, (c) Informations sur les PCBs en fonction du degré d'instruction. 


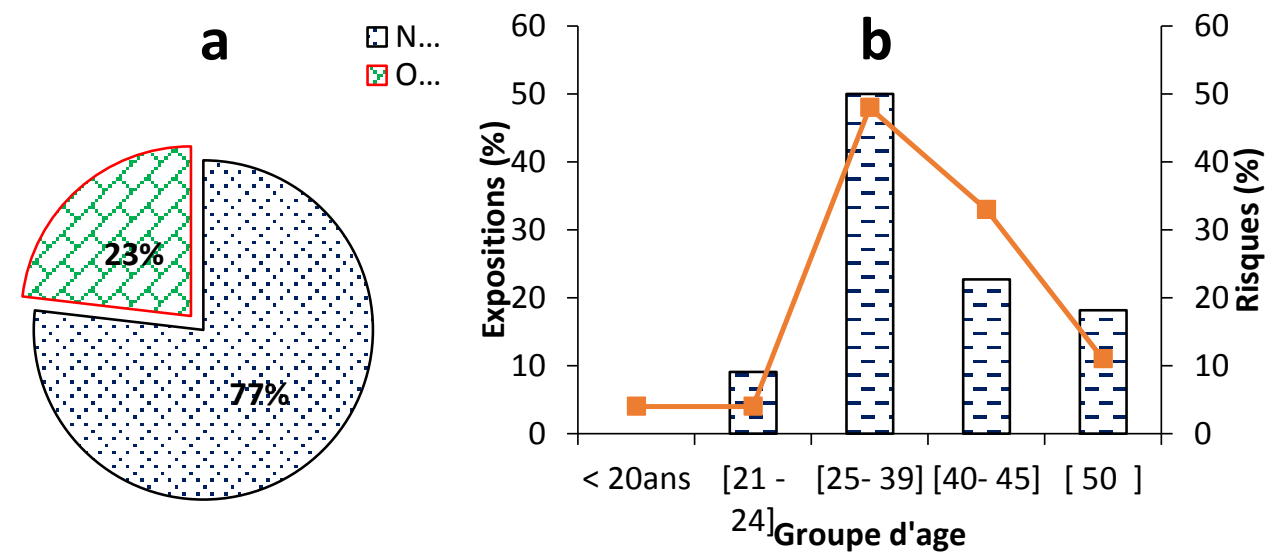

Figure 4: (a) Information générale relative à une possible exposition des aliments aux PCBs, (b) Constat d'exposition et risques aux PCBs en fonction de groupe d'âge.

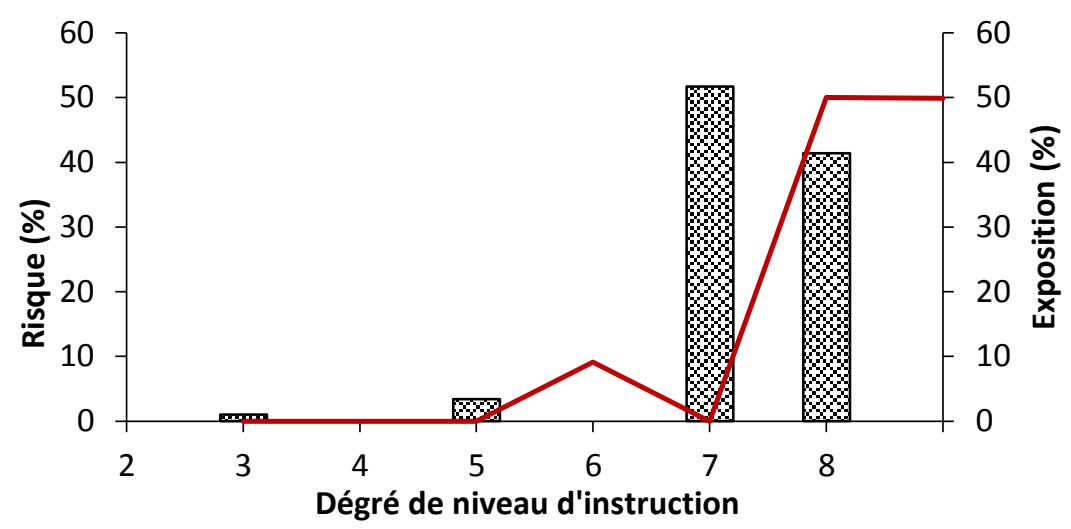

Figure 5: Exposition alimentaire constatée et risques liés aux PCBs en fonction de niveau d'instruction.

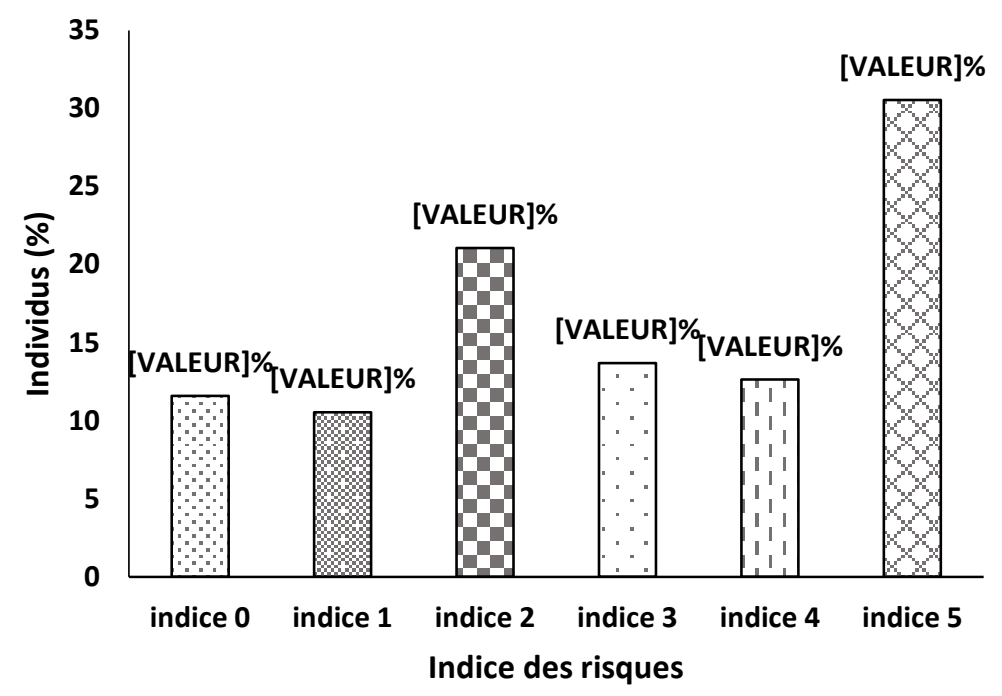

Figure 6: La proportion de la population en indice des risques liés aux PCBs. 


\section{DISCUSSION}

\section{Notion de pollution et information générale sur les PCBs}

Pour évaluer le niveau de connaissance des PCBs comme polluant contaminant dans un milieu à priori profane, il faut un identifiant perceptible par tous, car le propre de la pollution moderne est inaccessible aux profanes (Lao et al., 2018). C'est ainsi qu'un lien (réducteur quant à la source d'émission diverse des PCBs) est fait avec les transformateurs électriques des vielles installations urbaines. Les entretiens avec les différentes personnes interrogées ont permis de déceler les informations primaires d'ordre général sur la pollution. Ainsi, relativement à la notion de la pollution, à priori, la majorité ont une connaissance superficielle liée plus aux sens, or ce n'est pas tout polluant que l'on peut voir et sentir particulièrement les micropolluants volatils comme les PCBs (Ratier et al., 2018). Lorsque le lien de la présence des PCBs dans l'environnement, a été fait avec l'une des sources d'émission comme les vieux transformateurs électriques (Figure 2), la focalisation a été plus nette pour les répondants, car longtemps après l'interdiction de leur utilisation, c'est dans ce système fermé (transformateur et condensteur) que les quantités les plus importantes des PCBs peuvent être retrouvées (Schwarzenbach, 2010).

Pour cette partie préliminaire importante, les résultats indiquent que $62 \%$ des enquêtés ont répondu oui (Fgure 3a). Mais cette notion de pollution et d'information sur les PCBs sont dépendantes de l'âge et de niveau d'instruction (Figure 3b et 3c). En effet, sur la Figure 3b, on remarque que, presque la moitié (48\%) de ceux qui ont une notion sur la pollution et une information d'ordre générale sur les PCBs sont âgés de 25 à 39 ans alors que le groupe d'âge [ $\geq 50$ ans] et celui $[\leq 20$ ans] représentent respectivement $7 \%$ et $10 \%$. Quant à la répartition de ces connaissances en fonction de niveau d'instruction (Figure. 3c), elle montre que $58 \%$ de ceux qui ont ces notions, ont fait des études supérieures contre 5\% de ceux qui ont le niveau du collège.
Il ressort de ces deux analyses que la perception du risque lié à la pollution d'une manière générale, et l'identification $d u$ contaminant en particulier sont dépendants du niveau d'instruction, de la maturité tout court (âge), mais aussi de l'expérience du vécu liée à l'activité (25-39 ans de la population active) (Erickson et Kaley II, 2011; Brunet, 2007); cela réconforte l'approche fonctionnaliste de la perception des risques (Lao et al., 2018) où la perception du risque est liée à l'expérience de l'individu, ses connaissances, bref sa personnalité et la situation du risque elle-même.

\section{La notion de la pollution et l'exposition des aliments aux PCBs}

Pour évaluer la compréhension de la population enquêtée par rapport à l'exposition chronique ou prolongée, conduisant à une contamination des aliments et des humains aux PCBs, les questions ont été plus simples et plus directes puisqu'il s'agit d'abord d'un constat des fuites des transformateurs dans les installations électriques urbaines ou périurbaines faisant parti du quotidien de la population enquêtée. Aussi, les témoignages d'accidents d'explosion des transformateurs en services ont été rapportés par plusieurs répondants. D'ailleurs, ces faits perceptibles par tous ont été toujours pris en comptes par les différents inventaires des PCBs dans le cadre de la gestion des déchets dangereux, ces dernières années (Zhang, 2010).

C'est autour de ces phénomènes d'émission des PCBs dans l'environnement que s'appuie les études de détection de ses substances dans les différentes matrices environnementales jusqu'aux denrées alimentaires dans les pays où ces questions ont été prises en compte plus tôt (Cangialosi et al., 2007). Mais dans notre cadre d'étude, ces recherches de traces ou des quantités des PCBs n'ayant pas été faites, ce sont ces témoignages et les observations sur le terrain qui permettront de faire des liens probables de la contamination, et d'alerter les opinions. Les réponses à ces questions par oui ou non, ainsi que certaines précisions quant aux 
observations des personnes interrogées ont relevé les distributions suivantes: À priori, l'état de l'information, de la compréhension et de l'observation de ces enquêtés montre que 23\% (Figure 4a) seulement ont la notion de la pollution et de l'exposition aux contaminants PCBs, proches de la source d'émission. Par contre, les $77 \%$ n'ont pas conscience de cette situation de risque même s'ils peuvent, par ailleurs, habiter proche de ces installations, ou ont été témoins d'un accident d'explosion, conduisant à une contamination de l'environnement immédiat.

En plus de cela, certains témoignages des personnes interrogées ont rapporté une explosion des transformateurs au-dessus des habitations, ce qui, même s'il n'a pas été source d'inhalation directe pourrait contaminer le sol environnent, milieu de vie et d'aire d'élevage de la volaille, par excellence. Cette situation constituerait une source de transfert des PCBs du sol vers le tube digestif des oiseaux ou des petits ruminants (Fournier et al., 2012; Lombardo and Peck, 2018). Pour le premier cas, ce sont les œufs qui seraient contaminés avec une proportion de $95 \%$ de biodisponibilité relative comme confirmer par les travaux de Fournier et al. (2012) et ceux de Lombardo and Peck (2018). Pour le second cas, c'est dans le lait de la chèvre laitière, par exemple, que s'y trouveraient les PCBs, dans l'ordre de $50 \%$ de biodisponibilité relative selon les résultats de la même étude. Faudrait-il noter aussi un lessivage du sol contaminé, qui drainerait ces PCBs vers des cours d'eau, contaminant ainsi la faune, la flore et les sédiments fluviaux et marins (Brunet, 2007). Ce phénomène de la pollution des eaux, vu les propriétés liposoluble et bioaccumulative des PCBs (Bodiguel, 2008; Oziolor et al., 2018) peut avoir des effets contaminants sur les produits halieutiques, en particulier les poissons qui sont les denrées alimentaires les plus contaminés en PCBs. Ce niveau de contamination mérite qu'on s'y attarde un peu. Parmi les analyses de contrôle de qualité des produits importés, l'analyse de la détection des PCBs dans la chair des poissons, aussi bien locales qu'importés estelle incluse? Ya- t-il un suivi de l'évolution de la dose en cas de détection?

Par ailleurs, si c'est une femme enceinte qui serait exposée de manière chronique ou prolongée, ce qui pourrait arriver à la suite des fuites permanentes ou d'explosion à côtés des habitations, cela pourrait faire transférer les PCBs à son enfant via le lait maternel (Sangely, 2010; Okes, 2017). Aussi, cette notion de la pollution et de l'exposition aux PCBs sont dépendantes de l'âge (Figure 4b) et du niveau d'instruction (Figure 5). On remarque ainsi que le groupe d'âge [25-39 ans] est le plus attentionné $(50 \%$ de ceux qui ont pu faire le constat) vis à vis de la question de la contamination, alors que celui de moins de 20 ans, le groupe 1, n'a pas du tout conscient de cette situation de risque. Quant à l'appréciation de cette situation relativement au niveau d'instruction, c'est l'échantillon au degré 7 d'instruction qui perçoit mieux l'exposition à partir de cette pollution pendant que les niveaux 2 et 4 n'y font aucun lien. Alors, comme pour le premier paramètre de la notion de pollution et d'information sur les PCBs, c'est l'approche fonctionnaliste de la perception du risque qui gouverne le comportement et la prise de conscience relativement à la situation du risque.

\section{La notion de la contamination et les risques liées aux PCBs}

Pour quantifier la notion de la contamination des aliments ou des personnes et les risques liés aux PCBs, l'ensemble des réponses données par chaque personne interrogée a été étudié. Ainsi, de la notion de la pollution et de l'information connue (indice 1et 2), en passant par l'exposition des aliments et des personnes (indice 3), pour arriver à la notion de la contamination des aliments ou des personnes ainsi que les risques liés aux PCBs (indice 4), il a été obtenue une mesure totale de risque d'indice 5 pour tout individu ayant donné des 
réponses convergentes vers la nocivité des PCBs aussi bien pour la santé humaine que pour l'environnement.

C'est ainsi que $12 \%$ de la population totale enquêtée sont à un indice zéro de risque, c'est-à-dire, ne savent pas ce qu'est une pollution, donc ne peuvent être ni à un niveau de la perception ni à celui de la compréhension du propre de la pollution moderne (Lao et al., 2018). À l'indice 1 et 2 de risque, se trouvent $11 \%$ de la population enquêtée, qui ont su ce qu'est la pollution sans détails, et ont une information d'ordre générale sur les PCBs à travers un identifiant (transformateur ou condensateur électrique). L'indice 3 de risque caractérise la proportion des enquêtés capables de constater une exposition des humains ou des aliments aux PCBs, par conséquent, cette proportion représente $21 \%$ (Figure 6). Enfin, ceux qui établissent le lien entre le transfert des PCBs de l'environnement vers l'homme directement ou via l'alimentation, en indiquant au moins un risque lié à cette contamination, représentent $31 \%$ de la population et sont aux indices 4 puis 5 de risque.

De plus, on remarque, comme pour les autres paramètres, que celui de la contamination et de la perception du risque est dépendant de l'âge et du niveau d'instruction (Figure $4 \mathrm{~b}$ et Figure 5). Ainsi, des $31 \%$ de la population qui ont la notion des risques liés aux PCBs, il ressort que $48 \%$ sont âgés de 25 à 39 ans, alors que les personnes interrogées, âgées de moins de 20 ans, et ceux dont l'âge est compris entre 21 et 24 ans, représentent $4 \%$ par groupe, soit un individu par groupe d'âge. Pendant ce temps, 33\% sont âgés de 40 à 45 ans, lorsque les personnes interrogées dépassant 50 ans représentent $11 \%$.

Quant au paramètre niveau d'instruction, il influe de manière non ordonnée sur la notion des risques liés aux PCBs. En effet, les personnes au niveau d'instruction $1 ; 2 ; 4$; et 6 n'ont aucune perception de risque lié aux PCBs, pourtant ces personnes n'ont pas le même niveau d'instruction. Si les personnes de niveau d'instruction 1 et 2 n'ont pas la perception des risques liés aux PCBs, dépendamment de leur instruction, ceux du niveau 4 et 6 ont un niveau comparativement plus élevé, qui ne le leur permettent cependant pas, d'avoir une perception des risques liés aux PCBs. Il en résulte de cette situation que le niveau d'instruction n'est pas un paramètre suffisant pouvant permettre une perception de risques liés aux PCBs.

\section{Conclusion}

Les résultats de cette étude ont permis de mettre en évidence la méconnaissance du contaminant $\mathrm{PCBs}$ et les risques liés à ce polluant, par une large frange de la population enquêtée. Quoique le désire de consommer une alimentation saine par une population fortement urbanisée, il manque pour cette population, l'éveil d'établir le lien du transfert des polluants vers les denrées alimentaires susceptibles de concentrer ces substances. Les paramètres d'âge, d'instruction et de catégories professionnelles sont dépendants de l'information sue et partagée relative aux polluants PCBs. Enfin, ces résultats peuvent être exploités, d'une part, par les sociétés et entreprises détentrices de ces déchets, d'autre part, par les services chargés de la gestion des déchets dangereux de manière générale, ainsi que les services chargés de la prévention sanitaire et la protection de la population. Ce serait, pour ce faire, la mise en place d'une stratégie de sensibilisation et d'information de la population qui reposerait sur les différents paramètres étudiés.

\section{CONFLIT D'INTERETS}

Les auteurs déclarent qu'ils n'ont aucun conflit d'intérêts.

\section{CONTRIBUTIONS DES AUTEURS}

AI et MMT ont conçu l'étude, interprété les résultats et écrit le manuscrit; MMOT et SSZ ont exécuté l'étude. AR a supervisé l'étude. 


\section{REMERCIEMENTS}

Les auteurs remercient M. Idi Sani Fari et frères pour l'opportunité donnée par leur institution d'accomplir ce travail.

\section{REFERENCES}

Andric SA, Tatjana S, Kostic Snezana M, Dragisic Nebojsa L, Andric Stanko S, Stojilkovic Radmila Kovacevic Z. 2000. Acute effects of polychlorinated biphenyl- containing and -free transformer fluids on rat testicular steroidogenesis. Env. Health Persp., 10(108): 955-959. https://www.ncbi.nlm. nih.gov/pmc/articles/PMC1240128/\#

Ayandele AA, Fagade OE, Adenegan AA. 2012. Effect of ammonium salts on the biodegradation of used transformer oil using locally isolated microorganisms. Agric. Biol. J. N. Am., 3: 131-139. DOI: 10.5251/abjna.2012.3.3.131.139

Bodiguel X.2008. Caractérisation et modélisation des processus de bioaccumulation des PCBs chez le merlu (Merluccius merluccius) du golfe $\mathrm{du}$ Lion. Doctorat d'université. Université Montpellier I, Montpellier, France, p. 216.

Brunet JF. 2007. Etat des lieux sur la pollution des fleuves par les PCB dans le monde. Actions des pouvoirs publics. Exemples de décontamination. BRGM/RP-55835-FR, p. 167. http://infoterre.brgm.fr/rapports/RP55835-FR.pdf

Cangialosi F, Intini G, Liberti L, Notarnicola M, Stellacci P. 2007. Health risk assessment of air emissions from a municipal solid waste incineration plant - A case study. DOI: 10.1016/j.wasman.2007.05.006

Direction de l'Environnement. 2017. Projet des Gestions des PCBs, Niger. Direction de l'Environnement.

Erickson MD, Kaley II RG. 2011. Applications of polychlorinated biphenyls. Env. Sci. Poll. Res., 18(2): 135-15. https://doi.org/10.1007/s11356-

Fournier A, Feidt C, Travel A, Le BIzec B, Marchand P, Jondreville C. 2012.
Relative bioavailability to laying hens of indicator polychlorobiphenyls present in soil, Chemos., 88: 300-306. https://doi.org/10.1016/j.chemosphere.20 12.02.041

Francis JJ, Johnston M, Robertson C, Glidewell L, Entwistle V, Eccles MP, Grimshaw JM. 2010. What is an adequate sample size? Operationalising data saturation for theory-based interview studies. Psy. Health, 25: 12291245.

DOI:

10.1080/08870440903194015

Hadnagy E, Gardner KH, Rauch LM. 2007. Dechlorination of polychlorinated biphenyls, naphthalenes and dibenzo-pdioxins by magnesium/palladium bimetallic particles. J. Env. Sci. Health Part A, 42(6): 685-695. http://dx.doi.org/10.1080/109345207013 26222

Imbeault NY, Powlowski JB, Colbert CL, Bolin JT, Eltis LD. 2000. Steady-state kinetic characterization and crystallization of a polychlorinated biphenyl-transforming dioxygenase. $J$. Biol. Chem., 275(17): 12430-12437. DOI: $10.1074 / j b c .275 .17 .12430$

Kerchich Y, Moussaoui Y, Scholl G, Eppe G. 2018. Determination of PCDD/Fs and dlPCBs in ash and particle samples generated by an incineration plant for hospital and industrial waste in Northern of Algeria. Atm. Poll. Res., http://hdl.handle.net/2268/219092

Lao Q, Jiao L, Chen F, Chen L, Sun X. 2018. Influential factors and dry deposition of polychlorinated biphenyls (PCBs) in atmospheric particles at an Isolated Island (Pingtan Island) in Fujian Province, China. Atmosphere, 9(2): 59. DOI: $10.3390 /$ atmos 9020059

Li YC. 2007 Detoxification of selected chloro-organics by oxidation technique using chelate modified fenton reaction. $\mathrm{PhD}$ thesis. University of Kentucky, Lexington (Royaume Uni), p. 191.

Lombardo JP, Peck JA. 2018. Effects of exposure to polychlorinated biphenyls during different periods of development 
on ethanol consumption by male and female rats. Env.l poll. (Barking, Essex: 1987), 236: $\quad 334-342 . \quad$ DOI: 10.1016/j.envpol.2018.01.072

Mei-Lin Y, Yueliang LG, Chen-chin H, Walter JR. 2000. Menstruation and reproduction in women with polychlorinated biphenyl (PCB) poisoning, long- term- follow- up interviews of the women from the Taiwan Yucheng cohort. Inter. J. Epidem., 29: 672-677. DOI: https://doi.org/10.1093/ije/29.4.672

Nwinyi OC. 2011. Enrichment and Identification of Askarel oil (PCB blend) degrading bacteria enriched from landfill sites in Edo State, Nigeria. Agric. Biol. J. N. Am., 2(1): 89-100. http://eprints.covenantuniversity.edu.ng/i $\mathrm{d} /$ eprint $/ 75$

Okes, NC. 2017. Conservation ecology of the Cape clawless otter, Aonyx capensis, in an urban environment (Doctoral dissertation, University of Cape Town). http://open.uct.ac.za/handle/11427/27353

Oziolor EM, Apell JN, Winfield ZC, Back JA, Usenko S, Matson CW. 2018. Polychlorinated biphenyl (PCB) contamination in Galveston Bay, Texas: Comparing concentrations and profiles in sediments, passive samplers, and fish. Env. Poll. (Barking, Essex: 1987), 236: 609-618. DOI: 10.1016/j.envpol.2018.01.086

Ratier A, Dron J, Revenko G, Austruy A, Dauphin CE, Chaspoul F, Wafo E. 2018. Characterization of atmospheric emission sources in lichen from metal and organic contaminant patterns. Env. Sci. Poll. Res., 1: 1-13. https://doi.org/10.1007/s11356-0171173-x
Sangely M. 2010. Dégradation biologique des polychlorobiphényles. Génie des procédés et de l'environnement. Université de Toulouse, Toulouse (France), p. 246.

Schwarzenbach RP, Egli T, Hofstetter TB, von Gunten U, Wehrli B. 2010. Global water pollution and human health. Ann. Rev. Env. Res., 35: 109-136. https://doi.org/10.1146/annurev-environ100809-125342

Sopaj F. 2013. Study of the Influence of Electrode Material in the Application of Electrochemical Advanced Oxidation Processes to Removal of Pharmaceutical Pollutants from Water. Science et Technique de l'Environnement. Université Paris-Est, Paris, France ; 183.

Tajik R, Asilian H, Khavanin A, Jonidi A, Eshrati B, Soleimanian A. 2012. Decomposition of Askarel Oil by Microwave Radiation and $\mathrm{H} 202 / \mathrm{TiO} 2$ Agents in order to reduce occupational hazards. Iranian Journal of Toxicology, 6(17): 660-667. http://ijt.arakmu.ac.ir/ article-1-134-en.html

Weber R, Gonser S, Köhler J, Körner W, Herold C, Haag R, Krapp M, Peichl L. 2017. Biomonitoring of polychlorinated biphenyls in Bavaria/Germany-longterm observations and standardization. Env. Science Poll. Res., 1: 1-11. DOI: $10.1007 / \mathrm{s} 11356-017-$ 1108-6

Zhang Y. Tang Z. R. Fu X. Xu Y. J. 2010. TiO2- graphene nanocomposites for gas-phase photocatalytic degradation of volatile aromatic pollutant: is $\mathrm{TiO}_{2}-$ graphene truly different from other TiO2 - carbon composite materials? ACS Nano, $\quad$ 4(12): $\quad$ 7303-7314. DOI: $10.1021 / \mathrm{nn} 1024219$ 